\title{
Relación entre la motivación y el uso de herramientas TIC por los profesores de los centros de idiomas en universidades españolas y europeas

\author{
Relationship between motivation and the use of ICT tools by teachers of \\ language centers in spanish and european universities
}

\author{
Galina Savitskaia \\ galina.savitskaia@gmail.com \\ Instituto de Idiomas \\ Universidad de Navarra \\ Pamplona, España
}

\begin{abstract}
Resumen- Las universidades, como uno de los sujetos de desarrollo de la sociedad, no pueden permanecer al margen del uso e influencia de las TIC, por lo que los cambios tecnológicos obligan a sus docentes a asumir el reto de involucrarse en el uso de dichas herramientas educativas con el objetivo de mejorar los resultados de aprendizaje. El presente trabajo pretende indagar en la relación entre la motivación docente, la formación de los profesores y el uso de las tecnologías de la información y de la comunicación (TIC), circunscribiendo dicha relación al marco de la enseñanza de idiomas dentro del espacio europeo de la educación superior. A través de encuestas a más de cien profesores de diecisiete centros y de su posterior análisis crítico, el estudio concluye que, en general, las TIC se encuentran cada vez más integradas en los métodos de enseñanza, pero que dichos métodos distan mucho de ser debidamente rentabilizados debido a la influencia de determinados factores de motivación/desmotivación, como se expondrá en las siguientes páginas.
\end{abstract}

Palabras clave: factores de motivación docente, herramientas TIC, universidad, enseñanza de idiomas

Abstract- Universities as one of the areas of development of society cannot remain outside the use and influence of ICT and technological changes require teachers to take up the challenge and get involved in the use of educational ICT tools with the aim of improve learning outcomes. This study aims to investigate the relationship between teacher motivation and teacher training and use of information technologies and communication in the context of language teaching in the European higher education area by the apparent growth of the importance of technology today. Through surveys of more than 100 people from 17 centers and critical analysis, the study concludes that, in general, ICTs are increasingly integrated into teaching methods, but these are far from the most of them under the influence of motivational factors, as will be shown in the following pages

Keywords: teacher motivation factors, ICT tools, university, language

\section{INTRODUCCIÓN}

Hoy en día, los docentes han visto modificada su praxis educativa respecto a la ejercida en los mismos ámbitos de docencia de hace sólo una década, y que esta modificación viene dictada, en gran medida, por la aplicación de las nuevas tecnologías de la información y la comunicación (TIC) en el aula. Teniendo en cuenta este nuevo escenario, el objetivo de la presente investigación es averiguar en qué medida el uso de dichas herramientas TIC por parte del profesorado depende de factores de motivación, entendiendo como factores de motivación los estímulos que recibe el profesor, tanto internos - las características propias de docencia- como externos -las características propias del ámbito laboral-, y que le guían, de mejor o peor manera, en el desempeño de su labor docente. Estos factores de motivación puede serán además motivacionales si el estímulo es positivo, o desmotivacionales, si el estímulo es de signo negativo.

Aunque serán citados más adelante y con más exhaustividad, algunos de los factores motivacionales y desmotivacionales son los siguientes:

- motivacionales: optimización de la labor docente.

- fomento del interés de los alumnos por el idioma.

- promoción profesional dentro de la institución.

- cumplimiento de las normas de la institución.

- desmotivacionales: - sobrecarga lectiva.

- falta de conocimiento de las herramientas.

- falta de apoyo técnico por parte de la institución.

En este marco, este trabajo investiga y analiza los factores motivacionales y desmotivacionales para el uso de las herramientas TIC por parte de profesores de diecisiete Centros de Idiomas de diferentes universidades españolas y europeas - todos ellos Centros miembros de la Confederación Europea de Centros de Idiomas de la Educación Superior (CercleS).

Realmente se considera que el presente trabajo puede aportar ideas o líneas de investigación futuras relacionadas con la motivación de los docentes para el uso de las herramientas TIC en el ámbito laboral universitario y ofrecer propuestas para los departamentos de innovación educativa.

\section{CONTEXTO}


Dada la especial contribución de los conocimientos de las lenguas a la cohesión social y económica, las autoridades de la Unión Europea demuestran un interés constante por potenciar la enseñanza-aprendizaje de los idiomas extranjeros. Así, desde la Declaración de Bolonia (1999) y la propuesta de creación de un Espacio Europeo de Educación Superior (EEES), dicho interés ha transcendido al mundo universitario. Por otra parte, los cambios tecnológicos y el desarrollo de las herramientas educativas están transformando la enseñanzaaprendizaje de las lenguas extranjeras. El uso de las Tecnologías de la Información y de la Comunicación (de aquí en adelante TIC) impulsa el aumento de la interacción y colaboración entre estudiantes y profesores más allá de los espacios físicos del aula, tal y como nos lo señalan Buyse y Fonseca-Mora en "Technologies and second languages" (2016, p.2) cuando mencionan que, en el mundo globalizado, el uso de las tecnologías y la capacidad de comunicarse en una o varias lenguas han aumentado las posibilidades de comunicación entre las personas lo que, en cuanto a la enseñanza de idiomas se refiere, implica que un uso correcto de los medios digitales puede favorecer el aprendizaje autónomo del alumnado, tanto dentro como fuera del aula, pero siempre con la guía de un profesor.

Las instituciones de educación superior, conscientes de este nuevo contexto, han implantado en los nuevos planes europeos de educación en idiomas la inclusión de innovadoras propuestas metodológicas para el proceso de enseñanzaaprendizaje, muchas de ellas apoyadas en las TIC. Este proceso de actualización metodológico influye también al profesorado, quien en ocasiones se ve enfrentado a la necesidad de adquirir nuevas competencias y destrezas para el ejercicio de su actividad. El que las adquiera o no depende de su actitud, de su compromiso y del apoyo de la institución a la hora de satisfacer sus necesidades formativas y facilitar la incorporación de estos nuevos recursos.

En cuanto a la enseñanza de idiomas que es lo que nos ocupa en este trabajo, tiene completamente integrada la utilización de las TIC en las labores de docencia, pero también es verdad que esto no siempre supone ni una renovación pedagógica ni la convicción por parte del profesorado de su posible aplicación: "los métodos de enseñanza siguen siendo los mismos a los del siglo pasado, por lo que se requiere que su empleo genere innovación por parte del profesor en el nivel universitario" (Cerbian de la Serna, 2003, p. 28).

De esta forma, la evolución educativa origina que uno de los actores principales del proceso -el profesor- sea impulsado a adquirir y desarrollar competencias tecnológicas que le permitan estar actualizado y cubrir las demandas sociales, lo que da lugar a cambios sustanciales en su habitual forma de enseñar.

Habida cuenta de todo lo anteriormente mencionado, el objeto de este estudio es analizar los factores motivacionales y desmotivacionales, tanto internos -las características propias de docencia-, como externos -las características propias del ámbito laboral-, que influyen en el ámbito del trabajo docente en relación con la utilización de las TIC (recordamos brevemente que algunos de los factores motivacionales contemplados en este estudio son , entre otros, la optimización de la labor docente, el fomento del interés de los alumnos por el idioma o la promoción profesional dentro de la institución, mientras que dentro de los desmotivacionales contemplamos la sobrecarga lectiva, la falta de conocimiento de las herramientas o del apoyo técnico por parte de la institución.)

- $\quad$ El objetivo principal de esta investigación es explorar la relación existente entre la edad de los docentes, su formación en nuevas tecnologías, los factores de motivación, tanto intrínsecos como extrínsecos, y el uso de las herramientas TIC por parte de los profesores, específicamente profesores del Instituto de Idiomas de la Universidad de Navarra y de los Centros de Idiomas de algunas universidades españolas y europeas miembros de CercleS.

- Los objetivos específicos, siguiendo en esta dirección, son los siguientes:

- Relacionar la edad de los docentes con la formación en TIC.

- Analizar el uso realizado por los docentes de las diferentes herramientas educativas TIC. Como ya se ha mencionado anteriormente, las herramientas se dividen en tres grupos según sean utilizadas para el desarrollo de contenidos; la información, comunicación y colaboración; o la evaluación del alumnado.

- Valorar, dentro de cada grupo de herramientas TIC, en qué grado diferentes los factores motivacionales influyen positivamente en su utilización.

- Valorar, dentro de cada grupo de herramientas TIC, en qué grado los diferentes factores desmotivacionales influyen negativamente en su utilización.

- Comparar los datos obtenidos de las tres muestras: la del Centro de Idiomas en la Universidad de Navarra, las de otras universidades españolas y las de universidades europeas.

- Generar posibles buenas prácticas derivadas del análisis de las informaciones recabadas en los diferentes centros para que sean aplicadas por los mismos en la mejora de la práctica docente.

\section{DESCRIPCIÓN}

Atendiendo a los aspectos metodológicos y de diseño de la investigación se propone llevar a cabo un estudio que permita conocer y analizar los factores de motivación/desmotivación que potencian o limitan el uso de las herramientas TIC para el aprendizaje-enseñanza en los Centros de Idiomas universitarios.

En la investigación participaron ciento seis profesores de diecisiete Centros de Idiomas en las universidades europeas.

- $\quad$ Universidad de Navarra: 23 profesores

- $\quad$ Universidades españolas (9): 56 profesores

- $\quad$ Universidades europeas (7): 27 profesores

Para ello se realizan las encuestas cuantitativas a través de las cuales se recoge la información de tres muestras mediante un cuestionario de preguntas y respuestas múltiples que permiten su cuantificación y el tratamiento estadístico.

Como ya se ha explicado con anterioridad, el presente trabajo plantea la necesidad de conocer los efectos que tienen los factores de motivación/desmotivación en el empleo de nuevas tecnologías en forma de herramientas TIC en los profesores de idiomas. 
Partimos de la base de que estas herramientas apoyan el modelo lingüístico de adquisición de segundas lenguas como acción y comunicación ya que, como hemos apuntado, aunque la acción comunicativa sólo se produce en ambientes naturales -algo que no puede reproducir totalmente Internet- los estudios han demostrado que las características de la realidad virtual que recrea Internet hacen de ésta una herramienta casi "natural” para el proceso de enseñanza-aprendizaje, puesto que la capacidad de simulación facilita el aprendizaje.

\section{Tabla 1}

La categorización de las herramientas TIC

\begin{tabular}{|c|c|c|}
\hline $\begin{array}{l}\text { Grupo 1. } \\
\text { Desarrollo de cont. } \\
\text { educativos ( c ) }\end{array}$ & $\begin{array}{l}\text { Grupo } 2 . \\
\text { Información, com. y } \\
\text { colaboración ( i ) }\end{array}$ & $\begin{array}{l}\text { Grupo } 3 . \\
\text { Evaluación (e) }\end{array}$ \\
\hline \begin{tabular}{l}
\multicolumn{1}{c}{ G1c1 } \\
Textuales: repositorios \\
de textos, generadores \\
de ejercicios
\end{tabular} & $\begin{array}{c}\mathbf{G 2 i 1} \\
\text { Correo electrónico }\end{array}$ & $\begin{array}{c}\text { G3e1 } \\
\text { Pruebas de nivel } \\
\text { online }\end{array}$ \\
\hline \begin{tabular}{l}
\multicolumn{1}{c}{ G1c2 } \\
Multimedia: video, \\
audio, animación
\end{tabular} & \begin{tabular}{l}
\multicolumn{1}{c}{ G2i2 } \\
Mensajería instan. \\
WhatsApp,Telegram \\
\end{tabular} & \begin{tabular}{l}
\multicolumn{1}{c}{ G3e2 } \\
Rúbricas, \\
e-portafolios \\
\end{tabular} \\
\hline $\begin{array}{c}\text { G1c3 } \\
\text { Recursos online: } \\
\text { diccionarios online, } \\
\text { repositorios de } \\
\text { contenidos digitales }\end{array}$ & $\begin{array}{l}\text { G2i3 } \\
\text { Grupos de discusión, } \\
\text { foros }\end{array}$ & $\begin{array}{c}\text { G3e3 } \\
\text { Cuestionarios y } \\
\text { formularios de } \\
\text { evaluación en } \\
\text { línea } \\
\end{array}$ \\
\hline \multirow[t]{6}{*}{\begin{tabular}{l}
\multicolumn{1}{c}{ G1c4 } \\
Iconográficos: \\
imágenes, líneas de \\
tiempo, posters... \\
\end{tabular}} & $\begin{array}{c}\text { G2i4 } \\
\text { Grupos en RRSS }\end{array}$ & \begin{tabular}{l}
\multicolumn{1}{c}{ G3e4 } \\
Diarios de \\
aprendizaje con \\
tutoría
\end{tabular} \\
\hline & \begin{tabular}{l}
\multicolumn{1}{c}{ G2i5 } \\
Blogs, \\
microblogging \\
(Twitter)
\end{tabular} & $\begin{array}{l}\quad \text { G3e5 } \\
\text { Tests / } \\
\text { exámenes a } \\
\text { través de EVA }\end{array}$ \\
\hline & $\begin{array}{c}\text { G2i6 } \\
\text { Comunidades de } \\
\text { buenas prácticas }\end{array}$ & \begin{tabular}{l}
\multicolumn{1}{c}{ G3e6 } \\
Pruebas \\
interactivas con \\
comp. lúdico \\
\end{tabular} \\
\hline & $\begin{array}{c}\text { G2i7 } \\
\text { Videoconferencias } \\
\text { (Skype, Hangouts...) }\end{array}$ & \begin{tabular}{l}
\multicolumn{1}{c}{ G3e7 } \\
Pruebas \\
por videoconfer.
\end{tabular} \\
\hline & $\begin{array}{c}\text { G2i8 } \\
\text { Trabajo por } \\
\text { proyectos } \\
\end{array}$ & \\
\hline & $\begin{array}{c}\text { G2i9 } \\
\text { Clase invertida } \\
\text { (flipped classroom) }\end{array}$ & \\
\hline
\end{tabular}

Una vez realizada esta categorización (Grupos 1,2 y 3) según los ámbitos de la actividad docente se compararon los factores motivacionales y desmotivacionales que influyen en el uso de las herramientas TIC por parte de los profesores de idiomas.

Para eso se elaboró un cuestionario estructurado que fue enviado a los responsables de formación docente y a los directores de Centros de Idiomas en universidades españolas y europeas, acompañados de una carta explicativa en español e inglés.

Se decidió que las preguntas incluidas en la herramienta de recolección de datos debían ser preguntas de opción múltiple: se dan opciones de respuesta y se pide seleccionar una de las alternativas dadas. Se aceptaba como premisa validada una respuesta positiva de $50 \%$ o más de los encuestados (como una mayoría simple).

Los profesores marcaron las herramientas de cada Grupo que se utilizan "mucho", "bastante", "poco" o "nada". Considerando que el uso se deriva de factores motivacionales, la siguiente pregunta fue sobre el nivel de importancia que adjudica el profesor a cada uno de los nueve factores motivacionales (FM). Por el contrario, se suponía que el desuso de las herramientas TIC está relacionado con los seis factores desmotivacionales propuestos (FD).

En la Fig. 1 se puede ver el esquema del proceso incluyendo la lista de factores motivacionales (FM) y desmotivacionales (FD):

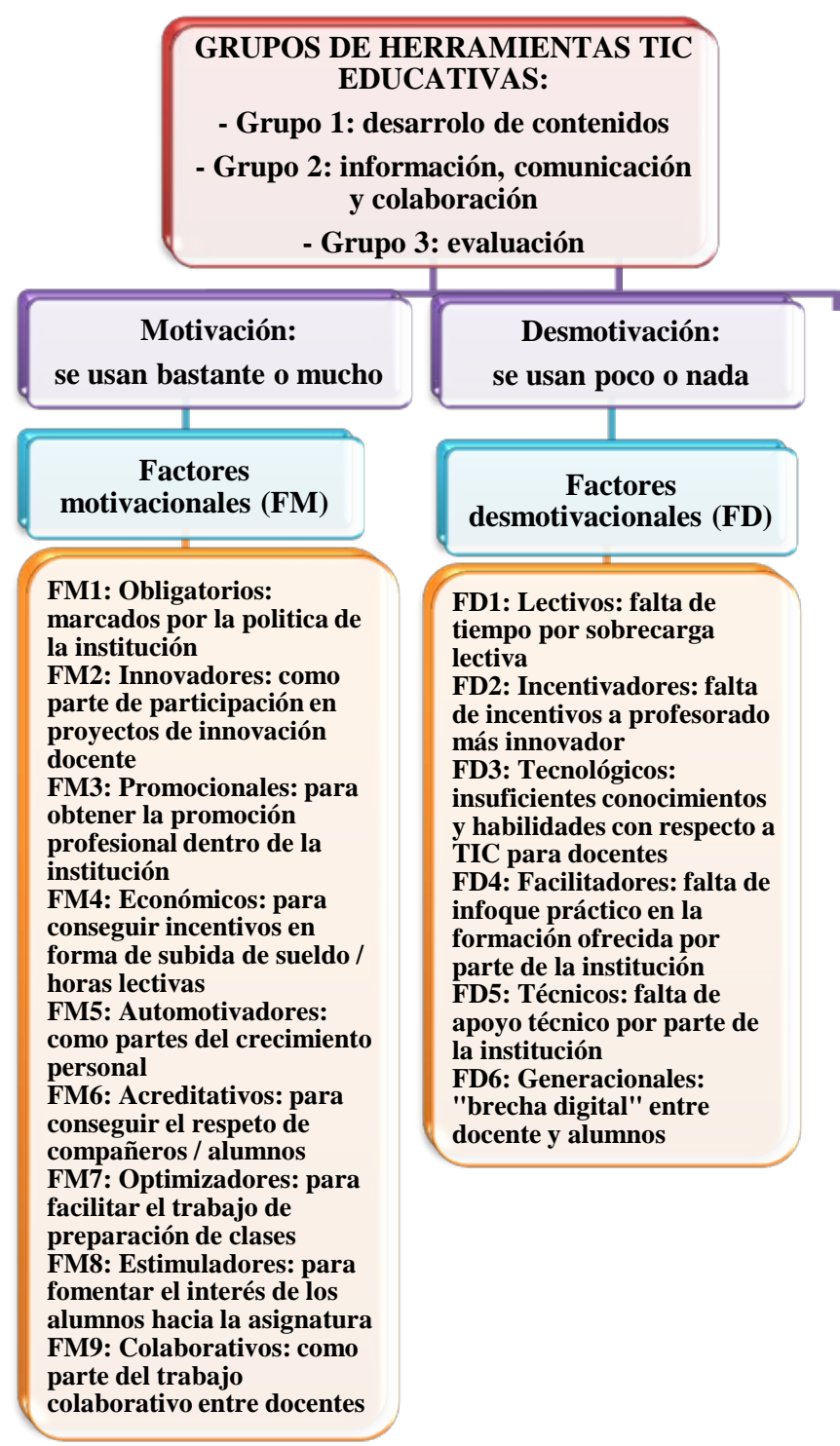

Figura 1: Factores motivacionales y desmotivacionales 
Dando esto por sentado, la investigación realizada a través de cuestionarios se centra en los siguientes aspectos:

- las primeras preguntas de cuestionario pretenden obtener la información sobre una posible relación entre la edad de los profesores de idiomas y la forma de obtener los conocimientos de TIC. Estas personas, que por su edad no son nativos digitales, curiosamente se han capacitado solos en el manejo de las herramientas TIC a través de estudios autodidactas (que al estar enfocados a las TIC obligatoriamente están "alojados" en manuales o tutoriales publicados en internet) o a través de cursos online y etc., que también presuponen un manejo de herramientas informáticas. En menor medida también señalan que han utilizado como recurso la formación ofrecida por las universidades. Basándonos en todo esto pudimos deducir que la edad de los profesores no dificulta el aprendizaje de herramientas TIC y que no les impide aprender TIC y desarrollar las habilidades necesarias para el uso de herramientas educativas.
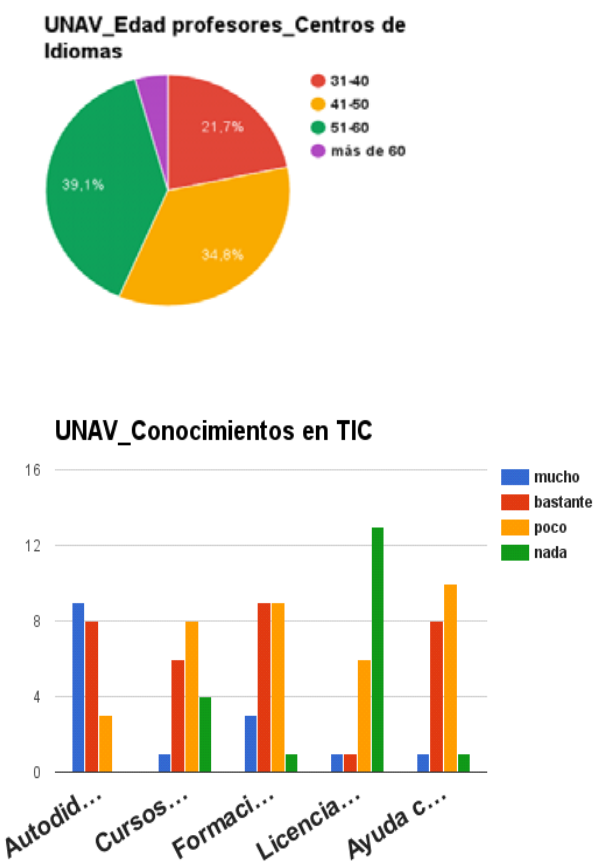

Figura 2: Ejemplo de resultados obtenidos

- $\quad$ las siguientes preguntas de cuestionario se basan en una división de las herramientas TIC en grupos o categorías según el uso en el proceso de enseñanza-aprendizaje.

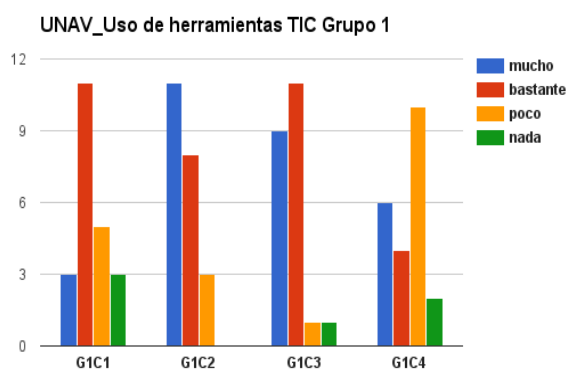

Figura 3: Ejemplo de resultados obtenidos para el uso de las herramientas TIC para el desarrollo de contenidos educativos por los profesores de idiomas de UNAV

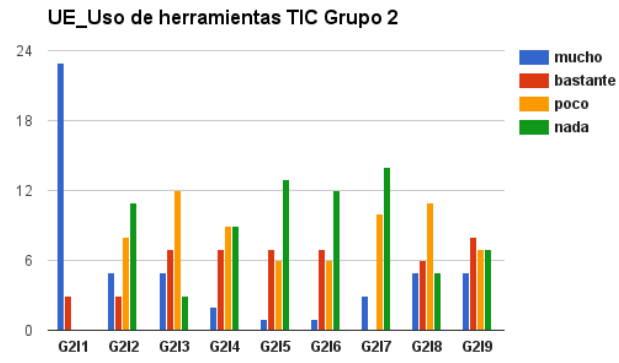

Figura 4: Ejemplo de resultados obtenidos para el uso de las herramientas TIC para la información y comunicación por los profesores de idiomas de las universidades europeas (CercleS)

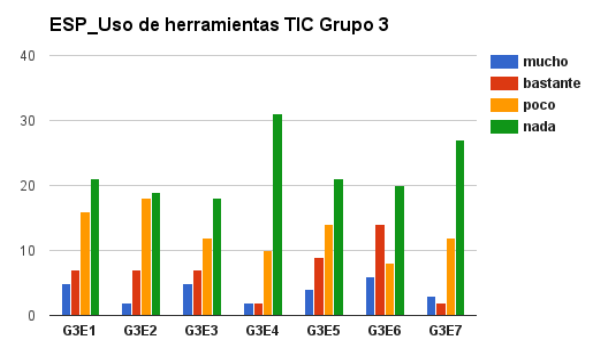

Figura 5: Ejemplo de resultados obtenidos para el uso de las herramientas TIC para evaluación educativa por los profesores de idiomas de las universidades españolas (CercleS)

\section{REsUltados}

Según los resultados obtenidos, los factores más importantes que motivan a los profesores de idiomas usar las herramientas TIC en su trabajo son claramente los siguientes:

- Automotivadores: como parte del crecimiento personal: FM5

- Optimizadores: facilitadoras del trabajo de preparación de clases: FM7.

- Estimuladores: fomento al interés de los alumnos hacia la asignatura: FM8

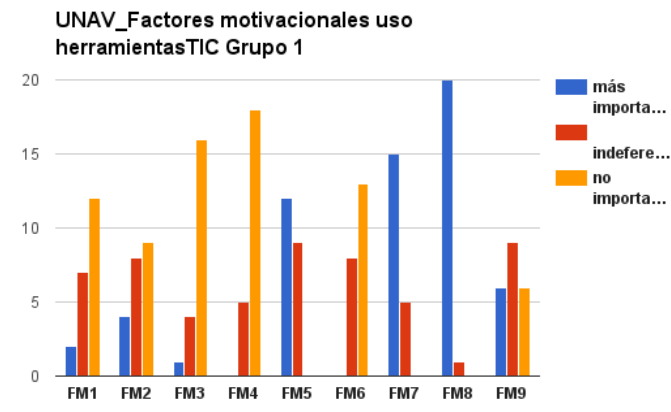

Figura 6: Ejemplo de resultados obtenidos

En cuanto a los factores que desmotivan a los profesores de idiomas para el uso las herramientas TIC en su trabajo, los más importantes son los siguientes: 


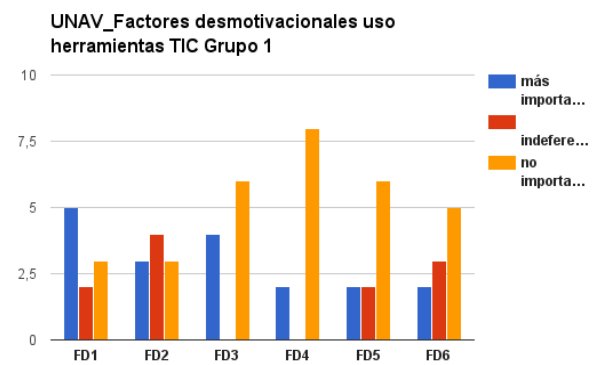

Figura 7: Ejemplo de resultados obtenidos

El dicho resultado coincide con los estudios recientes que indican que muchos profesores manifiestan que, frente a las clases tradicionales, la utilización de nuevas tecnologías requiere más tiempo (para la búsqueda de información y material, así como para la planificación y organización), y que no disponen de ese tiempo en sus "horarios de clase sobrecargados”.

- Insuficientes conocimientos y habilidades en TIC: FD3.

Otros profesores señalan que no han recibido formación suficiente para acceder a las tecnologías, y que por tal motivo no las utilizan. Sin embargo, siguiendo a Osborne \& Hennessy (2003) es muy interesante destacar la diferencia que los estudios resaltan entre la formación de tipo técnico (para utilizar los recursos), y la formación de tipo pedagógico (para aplicarlos en la enseñanza) y cómo es necesario "no sólo mejorar la formación del profesorado, sino adecuarla a sus necesidades docentes” (p. 5).

Además, se puede subrayar que los profesores de la Universidad de Navarra también marcaron como factor desmotivacional:

- $\quad$ Falta de incentivos para profesorado innovador: FD2

- $\quad$ Falta de apoyo técnico por parte de la institución: FD5

En atención a lo señalado, hay que comentar un hecho interesante que fue descubierto en el proceso de investigación; si los factores motivacionales más importantes son prácticamente los mismos para los profesores de los Centros de Idiomas españolas y europeas, los factores desmotivacionales que impiden el uso de las herramientas TIC son importantes solamente para los profesores de los Centros de Idiomas en las universidades españolas. Los profesores de las universidades europeas consideran que no existen factores desmotivacionales que puedan influir en el uso de las herramientas TIC educativas.

\section{CONCLUSIONES}

La integración de las Tecnologías de la Información y de la Comunicación en el proceso de enseñanza-aprendizaje de idiomas extranjeros en la educación superior es, actualmente, un hecho apoyado por varios proyectos de la Unión Europea. Muchas universidades en España y Europa disponen de Centros de Idiomas equipados con las EVA -entornos virtuales de aprendizaje-, conformados por un conjunto de herramientas informáticas que posibilitan la interacción didáctica considerada como una tecnología para crear y desarrollar cursos o modelos de formación didácticos en la web, pero que requieren tener conocimientos y habilidades en TIC por parte de los profesores y alumnos.

La conclusión final de este estudio, que pretende establecer la relación entre los factores de motivación de los docentes y el uso de los TIC, permite confirmar que la motivación intrínseca (personal) es la que más influye en el deseo de los profesores a la hora de innovar para prestar la mejor calidad de enseñanza-aprendizaje de idiomas extranjeros, en este caso, en forma de incorporación de las herramientas TIC en el patrón metodológico. Lo que se refiere a la motivación extrínseca (ámbito laboral), el estudio demuestra que las universidades, sobre todo las españolas, no utilizan toda su capacidad en este campo y que el profesorado se resiente de esta carencia. La motivación de los profesores para el uso de las nuevas tecnologías está directamente relacionada con un compromiso de la universidad para incorporar mejoras en el ámbito laboral, gracias a cuales el proceso de enseñanza-aprendizaje puede ascender a otro nivel.

Desde la perspectiva de este trabajo, se puede suponer que los docentes no necesitan ni pretenden ser especialistas en TIC, sino que buscan adquirir habilidades y conocimientos para mejorar su desempeño. Les motiva que los alumnos logren sus metas gracias a su trabajo diario y se dan cuenta de que las herramientas TIC se han convertido en un medio imprescindible para conseguir dichas metas.

Por otra parte, no podemos olvidar el aspecto motivador del ámbito laboral, donde tiene que promoverse la cultura de cooperación y coordinación. Con el fin de conseguirlo, los departamentos de innovación de las universidades o Centros de Idiomas deben prestar la información adecuada y organizar la formación de docentes, que incluya cursos a nivel de usuario, prácticas educativas, propuestas y organización de las jornadas de intercambio de ideas sobre sus programas, materiales educativos y organización de las clases. El docente debe saber utilizar las plataformas de enseñanza y preparar material que pueda estar a disposición de sus estudiantes en espacios virtuales. En conclusión, hay que propiciar una tendencia general de innovación que trascienda a la actividad universitaria tanto en su organización como en el reconocimiento social de la función docente.

Tal vez aquí sería acertado realizar un estudio sobre cómo y en qué ayudan las universidades europeas a sus profesores, ya que estos no señalan ningún factor desmotivador importante en su labor docente; este estudio, además, podría culminar o complementarse con jornadas de intercambio de experiencias entre docentes, jornadas que han sido señaladas en las encuestas cualitativas como un factor motivacional.

Otra aplicación práctica que consideramos útil para optimizar la aplicación de las herramientas TIC en la enseñanza de idiomas se presenta como una sistematización de las herramientas TIC susceptibles de uso en "fichas" o "recetas" que ayuden, con un solo vistazo, a restringir el abanico de la elección.

Las “fichas" delimitarían las características de la TIC respondiendo a cuestiones como pueden ser las siguientes:
a) número de alumnos ideal para utilizarla:
b) nivel de idioma aconsejado para utilizarla: 
c) función destacada:

d) características de la función destacada (tomamos para seguir desarrollando el ejemplo la función comunicativa):

e) grado de dificultad en el manejo de la herramienta

\section{Tabla 2}

Ejemplo de una "ficha"

Herramientas TIC recomendables: .........

\begin{tabular}{l|l}
\hline $\mathrm{N}^{\circ}$ DE ALUMNOS: & Grupos de 5 a 10 \\
\hline NIVEL: & $\mathrm{B} 2$ \\
\hline $\begin{array}{c}\text { FUNCIÓN } \\
\text { DESTACADA: }\end{array}$ & Comunicación \\
\hline CARACTERÍSTICAS: & $\begin{array}{c}\text { Permite tanto foros como } \\
\text { comunicación individualizada }\end{array}$
\end{tabular}

\begin{tabular}{|c|c|}
\hline & $\begin{array}{l}\text { Permite insertar video } \mathrm{y} \\
\text { audio }\end{array}$ \\
\hline & \begin{tabular}{lrr}
\multicolumn{1}{c}{ Permite } & \multicolumn{2}{c}{ corrección } \\
gramatical & sobre & las \\
intervenciones & visible & para \\
todo el grupo & &
\end{tabular} \\
\hline \begin{tabular}{l}
\multicolumn{1}{c}{ GRADO DE } \\
DIFICULTAD DE \\
MANEJO
\end{tabular} & Bajo \\
\hline
\end{tabular}

Con esta "fichas", elaboradas sobre las TIC consideradas óptimas para la enseñanza de idiomas -no para la enseñanza en general-, se ayuda al docente a cribar el abanico de TIC según parámetros que ya conoce $\left(\mathrm{n}^{\circ}\right.$ de alumnos y nivel), se le orienta en qué y cómo puede trabajar con la herramienta y se le informa sobre su nivel de complejidad.

Consideramos que, a pesar de algunas de las dificultades encontradas en el proceso de recogida de datos (el primero, que los Centros de Idiomas universitarios no siempre tienen un número fijo de profesores en la plantilla puesto que este número depende del plan de estudios anual y de la demanda de idiomas en cada semestre: y segundo, que la encuesta se envió solamente a los Centros de CercleS donde imparten no menos de cuatro idiomas extranjeros y el número de alumnos por año supera las 3000 personas), las líneas de investigación propuestas son válidas y ajustadas a la realidad de la docencia de idiomas, y que repercutirían favorablemente en el desempeño de su enseñanza.

\section{REFERENCIAS}

Adell Segura, J. (1997). Tendencias en Educación en la Sociedad de las Tecnologías de la Información. Edutec: Revista electrónica de tecnología educativa. $\mathrm{n}^{07}$

Austin, A., \& Gamson, Z. (1983). New demands, heightened tensions. ASHE-ERIC higher education research report. Association for the study of higher education. Washington DC. Traducción propia de http://files.eric.ed.gov/fulltext/ED243397.pdf

Brown, J. (2001). Using Surveys in Language Programs. Cambridge University Press.

Buyse, Kris; Fonseca-Mora, M ${ }^{\mathrm{a} C a r m e n ; . ~(2016) . ~ T e c h n o l o g i e s ~}$ and second languages. Comunicar journal, N. 50, v. 25.

Cabrero, J. (2007). Las necesidades de las TIC en el ámbito educativo: oportunidades. Tecnología y Comunicación Educativas (TyCE), 4-19.

Castelsl, M., \& Cardoso, G. (2005). The network society from Knowledge to Policy. DC: Johns Hopkins Center for Transatlantic Relations.

Cerbian de la Serna, M. (2003). Enseñanza virtual para la innovación universitaria. Madrid: Narcea Ediciones.

Karsenti, T., \& Lira-Gonzalez, M. (2011). La importancia de la motivación y las habilidades computacionales de los futuros profesores en el uso de las TIC. Revista Iberoamericana de Educación Superior, 116-129.

O’Meara, K. (2003). Reframing Incentives and Rewards for Community Service-Learning and Academic Outreach. Journal of higher Education outreach and engagement., 201. Traducción propia de http://openjournals.libs.uga.edu/index.php/jheoe/article/vi ew/196/184

Osborne, J., \& Hennessy, S. (2003). Literature Review in Science Education and the Role of ICT: Promise, Problems and Future Directions. Recuperado el 1 de junio de 2016, de https://telearn.archives-ouvertes.fr/hal00190441 